\title{
Experimental Confirmation of $n$-scroll Hyperchaotic Attractors
}

\author{
Simin Yu \\ College of Automation \\ Guangdong University of Technology \\ Guangzhou 510090, China \\ Jinhu Lü \\ Key Laboratory of Systems and Control \\ Institute of Systems Science \\ Academy of Mathematics and Systems Science \\ Chinese Academy of Sciences \\ Beijing 100080, China \\ Email: jhlu@iss.ac.cn \\ Guanrong Chen
Department of Electronic Engineering
Guanrong Chen
Department of Electronic Engineering
City University of Hong Kong
Hong Kong, China
Email: gchen@ee.cityu.edu.hk

(1)

an experimental verification of hyperchaotic $3-$ and $4-$ scroll

Abstract-A systematic circuit design approach is proposed for experimental verification of hyperchaotic $2,3,4$-scroll attractors from a generalized Matsumoto-Chua-Kobayashi (MCK) circuit. The recursive formulas for system parameters are rigorously derived for improving the hardware implementation.

\section{INTRODUCTION}

Hyperchaos was first observed from a real physical system by Matsumoto, Chua and Kobayashi in [1]. Then, Yalcin et al. [2] introduced some hyperchaotic $n$-double-scroll chaotic attractors by adding breakpoints in the piecewiselinear (PWL) characteristic of the MCK circuit and confirmed the hyperchaotic $4-$ and $6-$ scroll attractors by computer simulations. Yu et al. [3] proposed hyperchaotic $n$-scroll attractors and realized hyperchaotic $3 \sim 10$-scroll attractors by computer simulations. Itoh et al. [4] investigated the impulsive synchronization of a hyperchaotic double-scroll attractor and its application to spread-spectrum communication systems. It has been known that it is generally difficult to implement multi-scroll chaotic and hyperchaotic attractors by a physical electronic circuit. Yalcin et al. [5] experimentally confirmed 3 - and 5-scroll chaotic attractors in a generalized Chua's circuit, while Zhong et al. [6] proposed a systematical circuitry design method for physically implementing up to as many as ten scrolls visible on the oscilloscope. Han et al. [7] constructed a double-hysteresis building block to physically realize a 9-scroll chaotic attractor. There are some other approaches reported in the literature for the design and circuit implementation of multi-scroll chaotic attractors [8-14]. It is generally quite difficult to physically build a nonlinear resistor having an appropriate characteristic with many segments. In this effort, Lü et al. [13] designed a novel circuit diagram to physically verify the multi-directional multi-scroll chaotic attractors. The main obstacle is that the device must have a very wide dynamic range [3,6], however physical conditions always limit or even prohibit such circuit realization [6]. Recently, Lü and Chen [14] reviewed the main advances of multi-scroll chaos generation.

In this paper, we describe the design of a novel block circuit diagram to experimentally confirm hyperchaotic nscroll attractors. This is the first time in the literature to report attractors. Moreover, the derived recursive formulas for system parameters provide a theoretical basis for physical realization of hyperchaotic attractors with a large number of scrolls.

The rest of the paper is organized as follows. In Section II, a general MCK circuit is briefly described. Then, a novel block circuit diagram is designed for hardware implementation of hyperchaotic 2,3,4-scroll attractors, and its dynamic equation is rigorously derived in Section III. Conclusions are finally drawn in Section IV.

\section{A GENERALIZED MCK CIRCUIT}

The dimensionless state equation of the hyperchaotic MCK circuit is described by [1]

$$
\left\{\begin{array}{l}
\frac{d x}{d \tau}=\alpha[g(y-x)-z] \\
\frac{d y}{d \tau}=\beta[-g(y-x)-w] \\
\frac{d z}{d \tau}=\gamma_{0}(x+z) \\
\frac{d w}{d \tau}=\gamma y
\end{array}\right.
$$

where $g(y-x)=m_{1}(y-x)+0.5\left(m_{0}-m_{1}\right)[\mid y-$ $x+1|-| y-x-1 \mid]$. When $\alpha=2, \beta=20, \gamma_{0}=$ $1, \gamma=1.5, m_{0}=-0.2, m_{1}=3$, system (1) has a hyperchaotic double-scroll attractor with Lyapunov exponents $\lambda_{1}=0.24, \lambda_{2}=0.06, \lambda_{3}=0, \lambda_{4}=-53.8$.

To generate hyperchaotic $n$-scroll attractors from (1), we first generalize the characteristic function $g(y-x)$, given in [3], as follows:

$$
\begin{aligned}
g(y-x) & =m_{N-1}(y-x)+ \\
& 0.5 \sum_{i=1}^{N-1}\left(m_{i-1}-m_{i}\right)\left(\left|y-x+x_{i}\right|-\left|y-x-x_{i}\right|\right) .
\end{aligned}
$$

The recursive formulas of positive switching points $x_{i}(i=$ 
$2,3, \cdots, N-1)$ can be easily deduced as follows:

$$
\left\{\begin{array}{l}
x_{2}=\frac{\left(1+k_{1}\right) \sum_{i=1}^{1}\left(m_{i}-m_{i-1}\right) x_{i}}{m_{1}-1}-k_{1} x_{1} \\
x_{3}=\frac{\left(1+k_{2}\right) \sum_{i=1}^{2}\left(m_{i}-m_{i-1}\right) x_{i}}{m_{2}-1}-k_{2} x_{2} \\
\vdots \\
x_{N-1}=\frac{\left(1+k_{N-2}\right) \sum_{i=1}^{N-2}\left(m_{i}-m_{i-1}\right) x_{i}}{m_{N-2}-1}-k_{N-2} x_{N-2}
\end{array}\right.
$$

where $m_{i}(0 \leq i \leq N-1)$ are the slopes of the segments and radials in various PWL regions, and $k_{i}=\frac{x_{i+1}-x_{i}^{E}}{x_{i}^{E}-x_{i}}(1 \leq$ $i \leq N-2)$, in which $x_{i}^{E}(1 \leq i \leq N-2)$ are the positive equilibrium points of $g(x)$.

To control the hyperchaotic signal into the region of the operational amplifier, we may assume that $x_{1}<1$. Here, we suppose that $x_{1}=0.5$. From (3), we determine the system parameters as follows: (i) when $N=2, m_{0}=-0.2$, $m_{1}=3$, system (1) with (2) has a hyperchaotic doublescroll attractor; (ii) when $N=3, m_{0}=3, m_{1}=-0.8$, $m_{2}=3, x_{2}=1.8333$, system (1) with (2) has a hyperchaotic 3 -scroll attractor; (iii) when $N=4, m_{0}=m_{2}=-0.7$, $m_{1}=m_{3}=2.9, x_{2}=1.5289, x_{3}=3.0239$, system (1) with (2) has a hyperchaotic 4-scroll attractor.

\section{CiRCUIT DESIGN AND IMPLEMENTATION}

In this section, a circuit diagram is constructed to experimentally verify the hyperchaotic $2,3,4$-scroll attractors. Also, the dynamic equation is rigorously derived from the circuit diagram shown in Fig. 1.

\section{A. Circuit diagram and its dynamic equation}

Fig. 1 shows the circuit diagram, where $N_{1}$ is the generator of the negative resistor $-R$, and $N_{R}$ is the multi-PWL function generator satisfying $I_{N}=f\left(v_{C_{2}}-v_{C_{1}}\right)$. All operational amplifiers are selected as Type TL082. The voltage of the electric source is $E=15 \mathrm{~V}$. Thus, the saturating voltages of the operation amplifiers are $E_{\text {sat }}=14.3 \mathrm{~V}$.

According to Fig. 1, the circuit equation is derived as follows:

$$
\left\{\begin{array}{l}
C_{1} \frac{d v_{C 1}}{d t}=f\left(v_{C 2}-v_{C 1}\right)-i_{L 1} \\
C_{2} \frac{d v_{C 2}}{d t}=-f\left(v_{C 2}-v_{C 1}\right)-i_{L 2} \\
L_{1} \frac{d i_{L 1}}{d t}=v_{C 1}+R i_{L 1} \\
L_{2} \frac{d i_{L 2}}{d t}=v_{C 2}
\end{array}\right.
$$

where $f\left(v_{C 2}-v_{C 1}\right)=G_{N-1}\left(v_{C 2}-v_{C 1}\right)+$ $0.5 \sum_{i=1}^{N-1}\left(G_{i-1}-G_{i}\right)\left(\left|v_{C 2}-v_{C 1}+E_{i}\right|-\left|v_{C 2}-v_{C 1}-E_{i}\right|\right)$

is a piecewise-linear characteristic function.

Comparing systems (1) with (4), we get the transformation relationship of parameters as follows:

$$
\left\{\begin{array}{l}
\tau_{0}=2 R C_{1}, \tau=\frac{t}{\tau_{0}}, \alpha=2, \beta=\frac{2 C_{1}}{C_{2}}=20 \\
\gamma_{0}=\frac{2 R^{2} C_{1}}{L_{1}}=1, \gamma=\frac{2 R^{2} C_{1}}{L_{2 i_{L 1}}}=1.5 \\
x=\frac{v_{C 1}}{V_{B P}}, y=\frac{v_{C 2}}{V_{B P}}, z=\frac{R i_{L 1}}{V_{B P}}, w=\frac{R i_{L 2}}{V_{B P}} \\
V_{B P}=1 V, G_{i}=m_{i} G(i=0,1,2, \cdots), G=\frac{1}{R} \\
g(y-x)=R f\left(v_{C 2}-v_{C 1}\right),
\end{array}\right.
$$

where $V_{B P}=1 V, \frac{1}{\tau_{0}}=\frac{1}{2 R C_{1}}$ is the time-scale transformation factor.

From (5), we have the parameters: $L_{1}=9 \mathrm{mH}, L_{2}=$ $6 m H, C_{1}=50 n F, C_{2}=5 n F, R=300 \Omega$. Then, we can get the theoretical values of the resistors based on the parameters given in Section II as follows:

(1) For hyperchaotic 2-scroll attractor:

$$
\left\{\begin{array}{l}
G_{0}=\frac{m_{0}}{R}=-0.67 m S, G_{1}=\frac{m_{1}}{R}=10 \mathrm{mS} \\
E_{1}=x_{1} V_{B P}, r_{1}=\frac{R_{12}}{R_{11}}=G_{1} R_{2}-1=1.00 \\
r_{2}=\frac{R_{22}}{R_{21}}=\frac{E_{s a t}}{E_{1}}=28.6 \\
r_{3}=\frac{R_{32}}{R_{31}}=\frac{r_{2}}{R_{2}\left(G_{1}-G_{0}\right)}-1=12.4
\end{array}\right.
$$

(2) For hyperchaotic 3-scroll attractor:

$$
\left\{\begin{array}{l}
G_{0}=\frac{m_{0}}{R}=10 m S, G_{1}=\frac{m_{1}}{R}=-2.7 m S \\
G_{2}=\frac{m_{2}}{R}=10 m S, E_{i}=x_{i} V_{B P}(i=1,2), \\
r_{1}=\frac{R_{12}}{R_{11}}=G_{2} R_{2}-1=1.00 \\
r_{2}=\frac{R_{22}}{R_{21}}=\frac{E_{s a t}}{E_{2}}=7.80 \\
r_{3}=\frac{R_{32}}{R_{31}}=\frac{r_{2}}{R_{2}\left(G_{2}-G_{1}\right)}-1=2.08 \\
r_{4}=\frac{R_{42}}{R_{41}}=\frac{E_{s a t}}{E_{1}}-1=27.60 \\
r_{5}=\frac{R_{52}}{R_{51}}=-\frac{1+r_{4}}{R_{2}\left(G_{1}-G_{0}\right)}-1=10.29
\end{array}\right.
$$

(3) For hyperchaotic 4-scroll attractor:

$$
\left\{\begin{array}{l}
G_{0}=\frac{m_{0}}{R}=-2.3 m S, G_{1}=\frac{m_{1}}{R}=9.7 \mathrm{mS} \\
G_{2}=\frac{m_{2}}{R}=-2.3 m S, G_{3}=\frac{m_{3}}{R}=9.7 \mathrm{mS} \\
E_{i}=x_{i} V_{B P}(i=1,2,3), r_{1}=\frac{R_{12}}{R_{11}}=G_{3} R_{2}-1=0.93 \\
r_{2}=\frac{R_{22}}{R_{21}}=\frac{E_{s a t}}{E_{3}}=4.73 \\
r_{3}=\frac{R_{32}}{R_{31}}=\frac{r_{2}}{R_{2}\left(G_{3}-G_{2}\right)}-1=0.97 \\
r_{4}=\frac{R_{42}}{R_{41}}=\frac{E_{s a t}}{E_{2}}-1=8.35 \\
r_{5}=\frac{R_{52}}{R_{51}}=-\frac{1+r_{4}}{R_{2}\left(G_{2}-G_{1}\right)}-1=2.90 \\
r_{6}=\frac{R_{62}}{R_{61}}=\frac{E_{s a t}}{E_{1}}=28.6 \\
r_{7}=\frac{R_{72}}{R_{71}}=\frac{r_{6}}{R_{2}\left(G_{1}-G_{0}\right)}-1=10.90
\end{array}\right.
$$

\section{B. Experimental observations}

TABLE I

THE RATIOS OF THE RESISTORS $r_{n}=\frac{R_{n 2}}{R_{n}}(1 \leq n \leq 7)$ AND THE NUMBER OF THE SCROLLS N

\begin{tabular}{c|c|c|c|c|c|c|c}
\hline$r_{1}$ & $r_{2}$ & $r_{3}$ & $r_{4}$ & $r_{5}$ & $r_{6}$ & $r_{7}$ & $\mathrm{~N}$ \\
\hline 1.00 & 28.60 & 12.40 & & & & & 2 \\
\hline 1.00 & 7.80 & 2.08 & 27.60 & 10.29 & & & 3 \\
\hline 0.93 & 4.73 & 0.97 & 8.35 & 2.90 & 28.60 & 10.90 & 4 \\
\hline
\end{tabular}

\section{TABLE II}

THE RESISTORS $R_{n 2}=r_{n} R_{n 1}(1 \leq n \leq 7)$ AND THE NUMBER OF THE SCROLLS N

\begin{tabular}{c|c|c|c|c|c|c|c}
\hline$R_{12}$ & $R_{22}$ & $R_{32}$ & $R_{42}$ & $R_{52}$ & $R_{62}$ & $R_{72}$ & $\mathrm{~N}$ \\
\hline $10 k$ & $286 k$ & $12.4 k$ & & & & & 2 \\
\hline $10 k$ & $78 k$ & $2.08 k$ & $276 k$ & $10.29 k$ & & & 3 \\
\hline $9.3 k$ & $47.3 k$ & $0.97 k$ & $83.5 k$ & $2.90 k$ & $286 k$ & $10.9 k$ & 4 \\
\hline
\end{tabular}

Let $R_{1}=100 \mathrm{k} \Omega, R_{2}=0.2 \mathrm{k} \Omega, R_{31}=R_{51}=R_{71}=$ $1 \mathrm{k} \Omega, R_{11}=R_{21}=R_{41}=R_{61}=10 \mathrm{k} \Omega$. By comparing Fig. 1 with system (1) under (2), we can calculate the resistors $R_{n 2}(1 \leq n \leq 7)$ as shown in Tables I and II. As seen from Fig. 1, when $K_{1}, K_{2}$ are switched on and $K_{3}, K_{4}$ are 


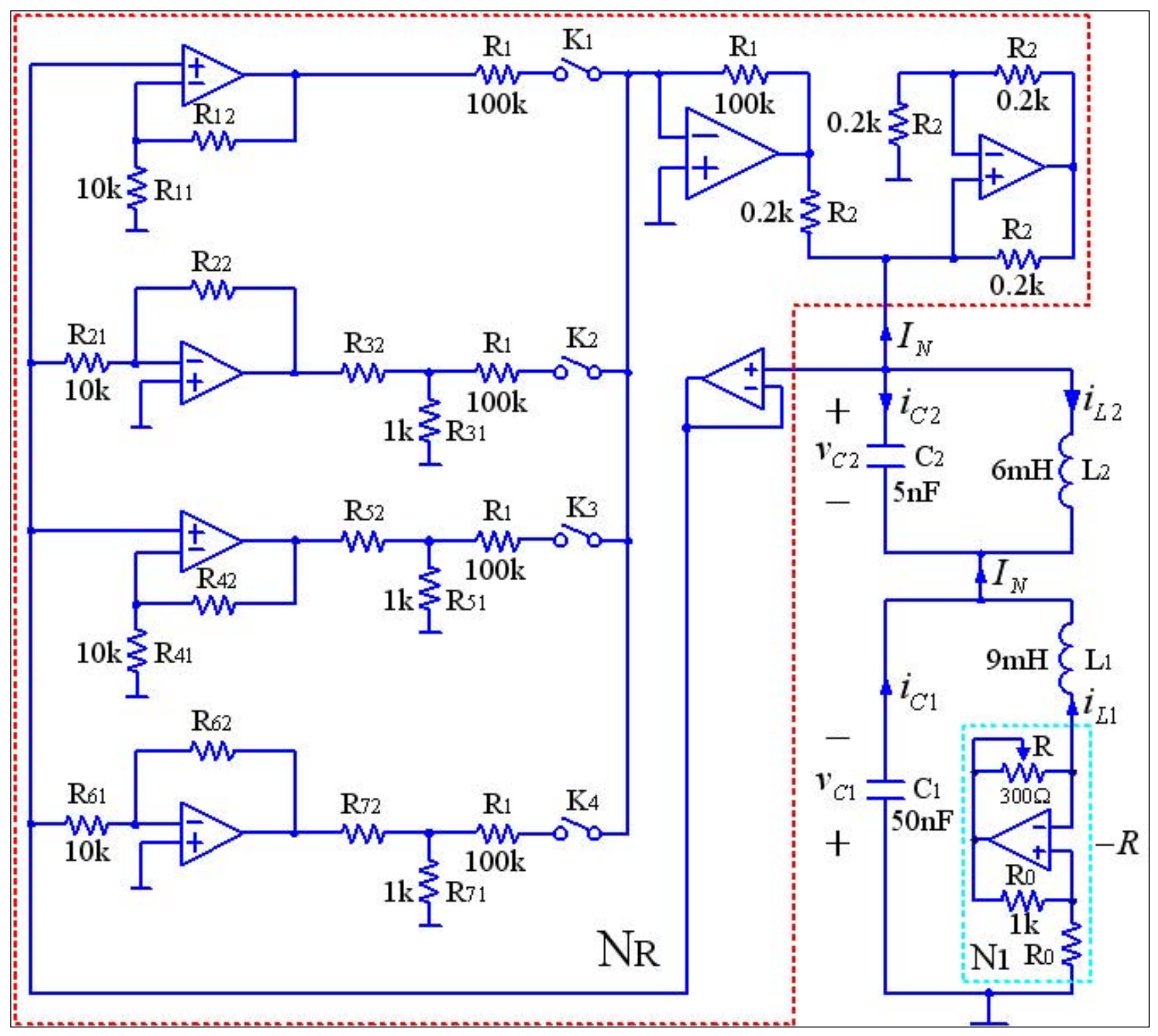

Fig. 1. Circuit diagram for generating hyperchaotic $n$-scroll attractors.

switched off, the circuit diagram can create a hyperchaotic double-scroll attractor; when $K_{1}, K_{2}, K_{3}$ are switched on and $K_{4}$ is switched off, the circuit diagram can generate a hyperchaotic 3-scroll attractor, as shown in Fig. 2 (a); when $K_{1}, K_{2}, K_{3}, K_{4}$ are switched on, the circuit diagram can create a hyperchaotic $4-$ scroll attractor, as shown in Fig. 2 (b).

\section{CONCLUSIONS}

This brief paper has proposed a novel block circuit diagram for hardware implementation of hyperchaotic 2,3,4-scroll attractors in a generalized MCK circuit. In addition, the derived recursive formulas for system parameters provide a theoretical basis for physical realization of the hyperchaotic attractors with a large number of scrolls.

\section{ACKNOWLEDGMENTS}

This work was supported by the National Natural Science Foundation of China under Grants No.60304017, No.20336040 and No.60572073, the Scientific Research Startup Special Foundation on Excellent $\mathrm{PhD}$ Thesis and Presidential Award of Chinese Academy of Sciences, Natural Science Foundation of Guangdong Province under Grants No.32469 and No.5001818, Science and Technology Program of Guangzhou City under Grant No.2004J1-C0291.

\section{REFERENCES}

[1] T. Matsumoto, L. O. Chua, and K. Kobayashi, "Hyperchaos: Laboratory experiment and numerical confirmation," IEEE Trans. Circuits Syst., vol. 33, no. 11, pp. 1143-1147, Nov. 1986. 


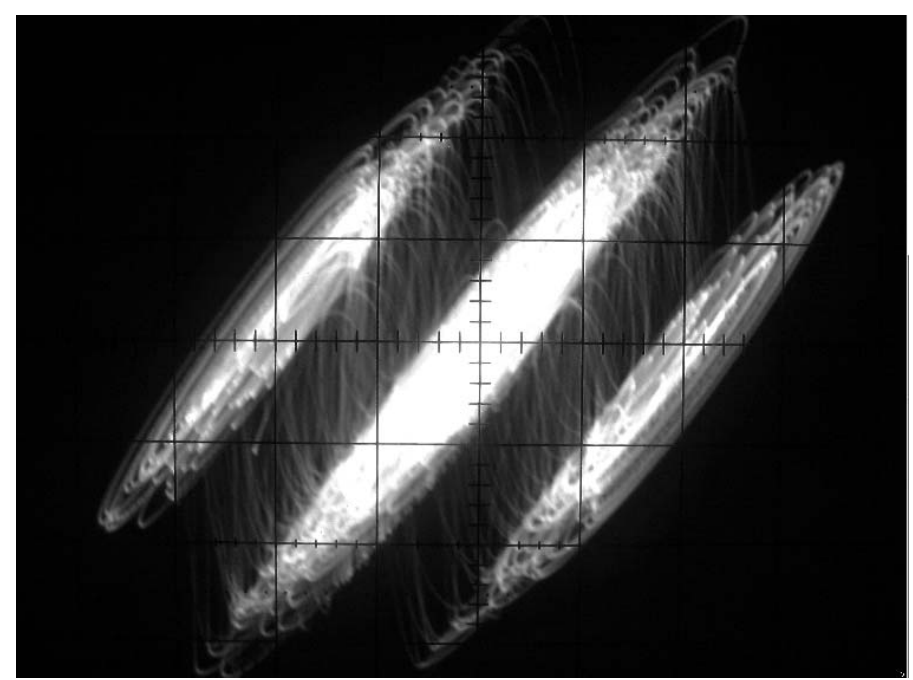

(a) 3-scroll

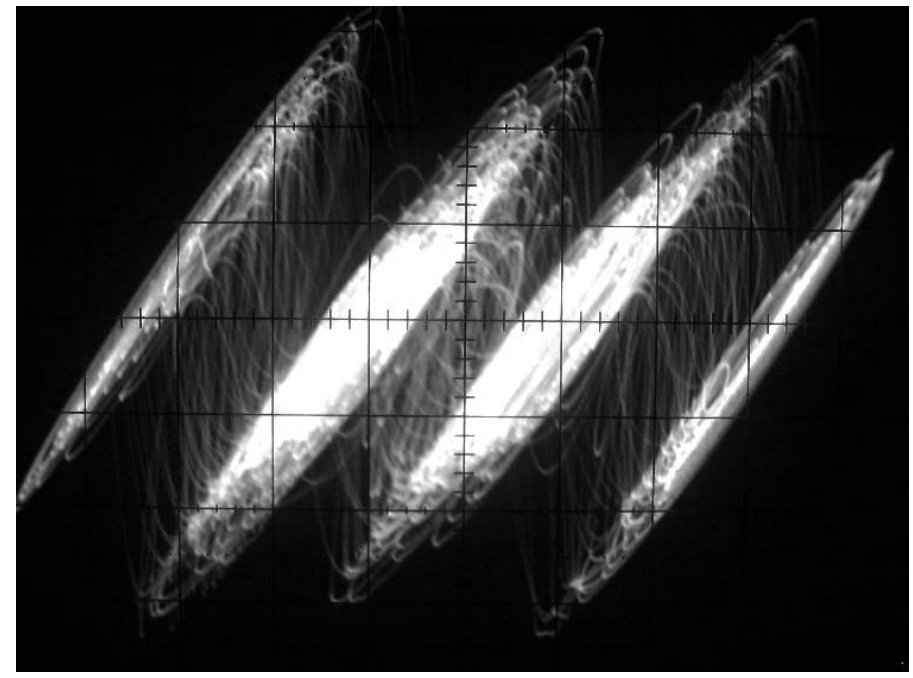

(b) 4-scroll

Fig. 2. Experimental observations of hyperchaotic $n$-scroll attractors.

[2] M. E. Yalcin, J. A. K. Suykens, and J. Vandewalle, "Hyperchaotic $n$-scroll attractors," Proceedings of the IEEE Workshop on Nonlinear Dynamics of Electronic systems (NDES 2000), Catania, Italy, pp. 25-28, 18-20 May 2000.

[3] S. M. Yu, Z. G. Ma, S. S. Qiu, S. G. Peng, and Q. H. Lin, "Generation and synchronization of $n$-scroll chaotic and hyperchaotic attractors in fourth-order systems," Chinese Physics, vol. 13, no. 3, pp. 317-328, Mar. 2004.

[4] D. A. Miller and G. Grassi, "Experimental realization of observer-based hyperchaos synchronization," IEEE Trans. Circuits Syst. I, vol. 48, no. 3, pp. 366-374, Mar. 2001.

[5] M. E. Yalcin, J. A. K. Suykens, and J. Vandewalle, "Experimental confirmation of 3- and 5-scroll attractors from a generalized Chua's circuit," IEEE Trans. Circuits Syst. I, vol. 47, no. 3, pp. 425-429, Mar. 2000.

[6] G. Zhong, K. F. Man, and G. Chen, "A systematic approach to generating $n$-scroll attractors," Int. J. Bifurcation Chaos, vol. 12, no. 12, pp. 29072915, Dec. 2002.

[7] F. Han, J. Lü, X. Yu, G. Chen, and Y. Feng, "Generating multi-scroll chaotic attractors via a linear second-order hysteresis system," Dynamics of Continuous, Discrete and Impulsive Systems Series B: Applications and Algorithms, vol. 12, no. 1, pp. 95-110, 2005.

[8] J. Lü, T. Zhou, G. Chen, and X. Yang, "Generating chaos with a switching piecewise-linear controller," Chaos, vol. 12, no. 2, pp. 344-
349, Jun. 2002.

[9] J. Lü, X. Yu, and G. Chen, "Generating chaotic attractors with multiple merged basins of attraction: A switching piecewise-linear control approach," IEEE Trans. Circuits Syst. I, vol. 50, no. 2, pp. 198-207, Feb. 2003.

[10] J. Lü, F. Han, X. Yu, and G. Chen, "Generating 3-D multi-scroll chaotic attractors: A hysteresis series switching method," Automatica, vol. 40, no. 10, pp. 1677-1687, Oct. 2004.

[11] J. Lü, G. Chen, X. Yu, and H. Leung, "Design and analysis of multiscroll chaotic attractors from saturated function series," IEEE Trans. Circuits Syst. I, vol. 51, no. 12, pp. 2476-2490, Dec. 2004.

[12] S. M. Yu, J. Lü, H. Leung, and G. Chen, "Design and implementation of $n$-scroll chaotic attractors from a general jerk circuit," IEEE Trans. Circuits Syst. I, vol. 52, no. 7, pp. 1459-1476, Jul. 2005.

[13] J. Lü, S. M. Yu, H. Leung, and G. Chen, "Experimental verification of multi-directional multi-scroll chaotic attractors," IEEE Trans. Circuits and Syst. I, vol. 53, no. 1, pp. 149-165, Jan. 2006.

[14] J. Lü and G. Chen, "Multi-scroll chaos generation: Theories, methods and applications," Int. J. Bifurcation Chaos, vol. 16, in press, 2006. 